\title{
THERMOELASTIC ANALYSIS OF PRESSURIZED HOLLOW SPHERICAL VESSELS WITH ARBITRARY RADIAL NON-HOMOGENEITY
}

\author{
Pooja RANI* and Kuldip SINGH \\ Department of Mathematics, Guru Jambheshwar University of Science and Technology \\ Hisar-125001, INDIA \\ E-mail: poojajindal742@gmail.com
}

\begin{abstract}
In this study, a general analysis of one dimensional steady-state thermal stresses of a functionally graded hollow spherical vessel with spherical isotropy and spherically transversely isotropy is presented with material properties of arbitrary radial non-homogeneity. The material properties may arbitrarily vary as continuous or piecewise functions. The boundary value problem associated with a thermo-elastic problem is converted to an integral equation. Radial and tangential thermal stress components distribution can be determined numerically by solving the resulting equation. The influence of the gradient variation of the material properties on the thermal stresses is investigated and the numerical results are presented graphically.
\end{abstract}

Keywords: functionally graded material; thermal stresses; radial non-homogeneity; pressure; spherical vessel.

\section{Introduction}

Functionally graded materials (FGMs) are composite materials which are designed to present a particular spatial variation of their properties. The "first" FGM was developed in Japan in 1984-1985 as the result of a space-plane project. FGMs are novel materials whose properties change gradually with respect to their dimensions. FGMs have the properties of the two raw materials which are mixed together and the component distribution is graded continuously. FGMs have obtained great attention of researchers in the past decade due to their graded properties at every single point in various dimensions. FGMs are widely used in engineering applications. FGMs can withstand very high thermal gradient, this makes them suitable for use in structures and space plane body, rocket engine component, etc.

Atkinson and Shampine [1] presented the algorithms and user interface of a MATLAB program, that solve numerically Fredholm integral equations (FIE) of the second kind on an interval $[a, b]$ to a specified, modest accuracy. One-dimensional steady-state thermal and mechanical stresses for a hollow thick sphere made of functionally graded material was discussed by Eslami et al. [2]. Guven and Baykara [3] considered a functionally graded isotropic hollow sphere with spherical symmetry subjected to internal pressure and this sphere was considered as a multimaterial sphere composed of a great number of concentric homogeneous spheres of different elasticity moduli. Li et al. [4] determined the elastic field of a functionally graded hollow spherical vessel with spherically isotropy and spherically transversely isotropy.

Nayak et al. [5] discussed the general analytical solution of a functionally graded thick spherical vessel with the assumption that the material properties vary with the power law of radius and Poisson's ratio remains constant. Assuming exponential-varying properties in the radial direction and based on the elasticity theory, an exact closed-form analytical solution to elastic analysis of FGM thick-walled cylindrical pressure vessels in the plane strain condition was obtained by Nejad et al. [6]. The boundary value problem was converted to a Fredholm integral equation to obtain the radial displacement and thermal stresses in a functionally graded hollow cylinder as in Peng and Li [7]. Rani et al. [8] studied thermal stresses of a functionally graded hollow

\footnotetext{
${ }^{*}$ To whom correspondence should be addressed
} 
thick cylinder due to non-uniform internal heat generation and obtained analytical solutions with radially varying properties by using the theory of elasticity.

Sahu et al. [9] presented a generalized solution for 1-D steady-state mechanical and thermal deformation and stresses in a rotating hollow functionally graded spherical body. Spherical shells were treated under mechanical and thermal loads in the form of rotational body force with heat generation. Sharma and Kaur [10] presented a numerical study of stress field in a functionally graded material hollow cylinder subjected to internal pressure and uniform heat generation. It was assumed that thermoelastic material properties of an FGM cylinder vary along the radius of the cylinder as an exponential function of the radius.

Yildirim [11] presented the thermo-mechanical analysis of a sphere made of non-homogeneous isotropic materials and proposed the closed form formulas for the elastic fields in a simple-power-law graded spheres subjected to steady-state thermal and internal/external pressure loads. You et al. [12] presented an accurate method for conducting elastic analysis of thick-walled spherical pressure vessels subjected to internal pressure.

In this study, we consider a functionally graded hollow spherical vessel with material properties of arbitrary non-homogeneity. The present research problem may find applications in the field of engineering. Thermo-elastic problem is converted to a Fredholm integral equation and we obtained the expressions for radial displacement and thermal stresses. Further solving the resulting equations, the distribution of radial and tangential thermal stress components have been obtained. The results of the thermal stresses are presented graphically. The obtained results coincide with the results of Li et al. [4] by neglecting the thermal effects and Nayak et al [5] for isotropic homogeneous material. The comparison of our results with Li et al. [4] and Nayak et al. [5] are also shown graphically in Section-4.

\section{Formulation of the problem}

Consider a spherical symmetrical problem made of a functionally graded material with an inner radius ' $a$ ' and outer radius ' $b$ ' that has arbitrarily varying material properties in the radial direction as shown in Fig.1.

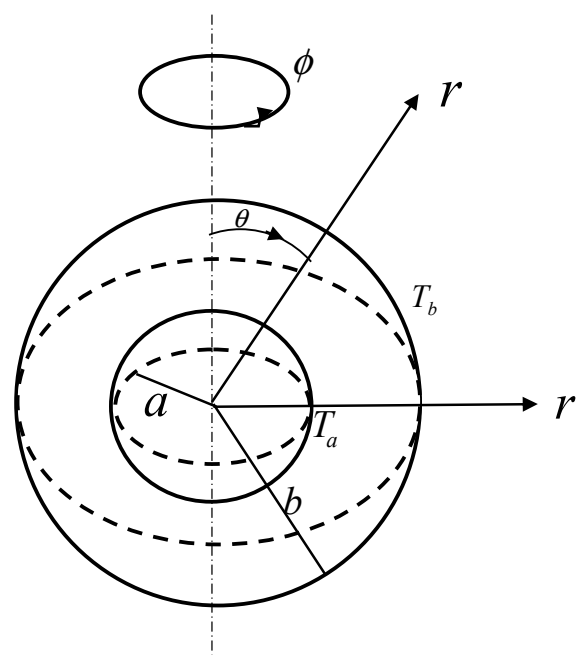

Fig.1 Functionally graded sphere.

\subsection{Temperature distribution}

To obtain desired thermal stresses in the functionally graded hollow sphere, it is natural to first determine the temperature distribution in the hollow sphere. To this end, the heat conduction equation without heat source for steady state is: 


$$
\frac{1}{r^{2}} \frac{d}{d r}\left[r^{2} k \frac{d T}{d r}\right]=0, \quad a \leq r \leq b
$$

and the thermal boundary conditions can be stated as

$$
C_{11} T(a)+C_{12} T^{\prime}(a)=f_{1}, \quad C_{21} T(b)+C_{22} T^{\prime}(b)=f_{2} .
$$

Here $T(r)$ is the temperature change, $k(r)$ is the thermal conduction coefficient, $C_{i j}(i, j=1,2)$ are the constant thermal parameters relative to the conduction and convection coefficient, $f_{j}(j=1,2)$ are known constants on the inner and outer radii, respectively.

In particular, two special cases of the above boundary conditions are that heat flux and temperature are prescribed. The former corresponds to $C_{j 1}=0$ and the latter corresponds to $C_{j 2}=0$.

By solving Eq.(2.1), we obtain

$$
T(r)=A \int_{a}^{r} \frac{1}{\rho^{2} k(\rho)} d \rho+B
$$

where A and B are constants, which can be determined using boundary conditions (2.2)

$$
\begin{gathered}
A=\frac{C_{11} C_{21} \int_{a}^{b} \frac{1}{\rho^{2} k(\rho)} d \rho+\frac{C_{11} C_{22}}{b^{2} k(b)}-\frac{C_{12} C_{21}}{a^{2} k(a)}}{}, \\
B=\frac{f_{1}\left[\int_{a}^{b} \frac{C_{21}}{\rho^{2} k(\rho)} d \rho+\frac{C_{22}}{b^{2} k(b)}\right]-\frac{C_{12}}{a^{2} k(a)} f_{2}}{C_{11} C_{21} \int_{a}^{b} \frac{1}{\rho^{2} k(\rho)} d \rho+\frac{C_{11} C_{22}}{b^{2} k(b)}-\frac{C_{12} C_{21}}{a^{2} k(a)}} .
\end{gathered}
$$

Here we consider two representative cases:

Case 1. The temperature change at the inner and outer surfaces:

For this case, taking $f_{1}=T_{a}, f_{2}=T_{b}$, using $C_{11}=C_{21}=1, C_{12}=C_{22}=0$ and the corresponding temperature distribution is

$$
T(r)=\frac{T_{b}-T_{a}}{\int_{a}^{b} \frac{1}{\rho^{2} k(\rho)} d \rho} \int_{a}^{r} \frac{1}{\rho^{2} k(\rho)} d \rho+T_{a} .
$$

Case 2. The temperature change at the inner surface is assumed to be prescribed as $f_{l}=T_{a}$ and heat flux at the outer surface is given as $f_{2}$ :

For this case, we have $C_{11}=C_{22}=1, C_{12}=C_{21}=0$ and the corresponding temperature distribution is 


$$
T(r)=b^{2} k(b) f_{2} \int_{a}^{r} \frac{1}{\rho^{2} k(\rho)} d \rho+T_{a},
$$

with these solutions of steady-state heat conduction, the thermal stresses in the hollow sphere can be determined in subsequent sections.

\subsection{Thermoelastic problem}

Consider a thermoelastic problem of such a non-homogeneous hollow spherical vessel made of functionally graded materials. Adopting a spherical coordinate system, the basic stress-strain relations are:

$$
\begin{aligned}
& \frac{d u_{r}}{d r}=\frac{1}{E}\left(\sigma_{r r}-2 v \sigma_{\theta \theta}\right)+\alpha T(r), \\
& \frac{u_{r}}{r}=\frac{1}{E}\left[(1-v) \sigma_{\theta \theta}-v \sigma_{r r}\right]+\alpha T(r) .
\end{aligned}
$$

where $E$ is the Young's modulus, $v$ is Poisson's ratio and $\alpha$ is the coefficient of thermal expansion. These are assumed to be depended on $r$ i.e.

$$
E=E(r), \quad v=v(r), \alpha=\alpha(r), \quad a \leq r \leq b .
$$

These quantities continuously vary in spatial direction for non-homogeneous media.

For a functionally graded hollow spherical vessel, the equilibrium equation, in the absence of body forces, can be stated as

$$
\frac{d \sigma_{r r}}{d r}+\frac{2\left(\sigma_{r r}-\sigma_{\theta \theta}\right)}{r}=0
$$

To obtain $\sigma_{r r}$ and $\sigma_{\theta \theta}$, we express them in terms of $u_{r}$ :

$$
\begin{aligned}
\sigma_{r r} & =\frac{E(r)}{[1-2 v(r)][1+v(r)]}\left[[1-v(r)] \frac{d u_{r}}{d r}+2 v(r) \frac{u_{r}}{r}\right]-\frac{E(r) \alpha(r) T(r)}{[1-2 v(r)]}, \\
\sigma_{\theta \theta} & =\frac{E}{[1-2 v(r)][1-v(r)]}\left[v(r) \frac{d u_{r}}{d r}+\frac{u_{r}}{r}\right]-\frac{E(r) \alpha(r) T(r)}{[1-2 v(r)]} .
\end{aligned}
$$

From Eq.(2.10) we get

$$
\frac{d u_{r}}{d r}+\frac{2 v(r)}{1-v(r)} \frac{u_{r}}{r}=\frac{[1+v(r)][1-2 v(r)]}{[1-v(r)] E(r)} \sigma_{r r}+\frac{[1+v(r)]}{[1-v(r)]} \alpha(r) T(r) .
$$

Solving this equation, we obtain 


$$
u_{r}=\frac{1}{\chi(r)}\left[C+\int_{a}^{r} \chi(s)\left\{\frac{[1+v(s)][1-2 v(s)]}{[1-v(s)] E(s)} \sigma_{r r}(s)+\frac{[1+v(s)]}{[1-v(s)]} \alpha(s) T(s)\right\} d s\right]
$$

where $\mathrm{C}$ is a constant to be determined through proper boundary conditions and $\chi(r)$ is defined by

$$
\chi(r)=\exp \left[\int_{a}^{r} \frac{2 v(s)}{[1-v(s)] s} d s\right]
$$

Solving Eqs (2.10)-(2.11),

$$
\sigma_{\theta \theta}=\frac{v(r)}{1-v(r)} \sigma_{r r}+\frac{E(r)}{1-v(r)} \frac{u_{r}}{r}-\frac{E(r) \alpha(r) T(r)}{1-v(r)} .
$$

Thus, once $\sigma_{r r}$ is determined, $u_{r}$ and $\sigma_{\theta \theta}$ can be obtained by Eqs (2.12) and (2.14), respectively. Clearly, the radial displacement $u_{r}$ is always continuous, even for piece-wise continuous $E(r)$ and $v(r)$. However, this is not true for tangential stress $\sigma_{\theta \theta}$, because it has a jump at the interface due to a mismatch of $E(r)$ and $v(r)$ at the interface of a multilayered structure. But we have considered a material with continuously varying properties.

Putting Eq.(2.12) into Eq.(2.14), then into Eq.(2.9), we get an integro-differential equation as follows:

$$
\begin{aligned}
& \frac{d \sigma_{r r}}{d r}+\frac{2[1-2 v(r)]}{r[1-v(r)]} \sigma_{r r}-\frac{2 E(r)}{r^{2} \chi(r)[1-v(r)]} \times \\
& \times\left[C+\int_{a}^{r} \frac{[1+v(s)][1-2 v(s)] \chi(s)}{[[1-v(s)] E(s)} \sigma_{r r}(s) d s\right]=F(r)
\end{aligned}
$$

where

$$
F(r)=\frac{2 E(r)}{r^{2} \chi(r)[1-v(r)]} \int_{a}^{r} \frac{[1+v(s)] \chi(s) \alpha(s) T(s)}{[1-v(s)] E(s)} d s-\frac{2 E(r) \alpha(r) T(r)}{r[1-v(r)]} .
$$

Integrating Eq.(2.15) with respect to $r$, we can get

$$
\sigma_{r r}(r)+\int_{a}^{r} K(r, \rho) \sigma_{r r}(\rho) d \rho=D+C f(r)+F_{l}(r)
$$

with

$$
\begin{aligned}
& K(r, \rho)=\frac{2[1-2 v(\rho)]}{\rho[1-v(\rho)]}-\frac{[1+v(\rho)][1-2 v(\rho)] \chi(\rho)}{[1-v(\rho)] E(\rho)} \int_{\rho}^{r} \frac{2 E(s)}{s^{2} \chi(s)[1-v(s)]} d s, \\
& f(r)=\int_{a}^{r} \frac{2 E(s)}{s^{2} \chi(s)[1-v(s)]} d s,
\end{aligned}
$$


and

$$
F_{l}(r)=\int_{a}^{r} F(s) d s
$$

where $K(r, \rho), f(r)$ and $F_{l}(r)$ in Eq.(2.17) are known and $C$ and $D$ are two unknown constants.

Furthermore, when considering a pressurized functionally graded hollow spherical vessel with inner and outer radii $a$ and $b$, respectively, the corresponding boundary conditions can be stated as

$$
\sigma_{r r}(a)=-q_{i} \quad \text { and } \quad \sigma_{r r}(b)=-q_{0}
$$

where $q_{i}$ and $q_{0}$ are two constants.

Using the boundary conditions in Eq.(2.17), C and D can be determined as

$$
C=\frac{q_{i}-q_{0}}{f(b)}-\frac{F_{l}(b)}{f(b)}+\frac{1}{f(b)} \int_{a}^{b} K(b, \rho) \sigma_{r r}(\rho) d \rho,
$$

and $D=-q_{i}$.

Now inserting above results in Eq.(2.17) and after solving, we get

$$
\sigma_{r r}(r)+\int_{a}^{b} L(r, \rho) \sigma_{r r}(\rho) d \rho=h(r)
$$

where

$$
L(r, \rho)= \begin{cases}K(r, \rho)-\frac{f(r)}{f(b)} K(b, \rho), & \rho<r, \\ -\frac{f(r)}{f(b)} K(b, \rho), & \rho>r,\end{cases}
$$

and

$$
h(r)=-q_{i}-\frac{F_{l}(b)}{f(b)} f(r)-\frac{q_{i}-q_{0}}{f(b)} f(r)+F_{l}(r) .
$$

\section{Transversely isotropic functionally graded sphere}

The results in the preceding section can be extended to a transversely isotropic functionally graded sphere. For this purpose, the equilibrium Eq.(2.9) remains unchanged, the only difference is that the following stress-strain relations for a spherically transversely isotropic material are used instead of those in Eq.(2.8):

$$
\begin{aligned}
& \frac{d u_{r}}{d r}=\frac{1}{E}\left(\sigma_{r r}-2 v \sigma_{\theta \theta}\right)+\alpha(r) T(r), \\
& \frac{u_{r}}{r}=\frac{1-v}{E^{*}} \sigma_{\theta \theta}-\frac{v}{E} \sigma_{r r}+\alpha(r) T(r) .
\end{aligned}
$$


Following the same procedure as in the preceding section, we have obtained the expressions for stresses and displacement as given below:

$$
\sigma_{r r}=\frac{E(r)}{\left[\lambda(r)-2 v^{2}(r)\right]}\left[\lambda(r) \frac{d u_{r}}{d r}+2 v(r) \frac{u_{r}}{r}-[\lambda(r)+2 v(r)] \alpha(r) T(r)\right]
$$

and

$$
\sigma_{\theta \theta}=\frac{E(r)}{\left[\lambda(r)-2 v^{2}(r)\right]}\left[v(r) \frac{d u_{r}}{d r}+\frac{u_{r}}{r}-[1+v(r)] \alpha(r) T(r)\right]
$$

where $\lambda(r)=[1-v(r)] \frac{E(r)}{E^{*}(r)}$, then we have

$$
u_{r}=\frac{1}{\chi(r)}\left[C+\int_{a}^{r} \chi(s)\left\{\frac{\left[\lambda(s)-2 v^{2}(s)\right]}{\lambda(s) E(s)} \sigma_{r r}(s)+\frac{[\lambda(s)+2 v(s)]}{\lambda(s)} \alpha(s) T(s)\right\} d s\right]
$$

where

$$
\chi(r)=\exp \left[\int_{a}^{r} \frac{2}{s}\left(\frac{\lambda(s)-v(s)}{\lambda(s)}\right) d s\right]=\exp \left[\int_{a}^{r} \frac{2}{s}\left(1-\frac{v(s) E^{*}(s)}{[1-v(s)] E(s)}\right) d s\right]
$$

Equation (3.2) can be written as

$$
\sigma_{\theta \theta}=\frac{v(r)}{\lambda(r)} \sigma_{r r}+\frac{E(r)}{\lambda(r)} \frac{u_{r}}{r}-\frac{E(r) \alpha(r) T(r)}{\lambda(r)}
$$

and from Eq.(2.9), we get

$$
\frac{d \sigma_{r r}}{d r}+\frac{2[\lambda(r)-v(r)]}{r \lambda(r)} \sigma_{r r}-\frac{2 E(r)}{r^{2} \chi(r) \lambda(r)} \times\left[C+\int_{a}^{r} \frac{\left[\lambda(s)-2 v^{2}(s)\right] \chi(s)}{\lambda(s) E(s)} \sigma_{r r}(s) d s\right]=F(r)
$$

where

$$
F(r)=\frac{2 E(r)}{r^{2} \chi(r) \lambda(r)} \int_{a}^{r} \frac{[\lambda(s)+2 v(s)] \chi(s) \alpha(s) T(s)}{\lambda(s) E(s)} d s-\frac{2 E(r) \alpha(r) T(r)}{r \lambda(r)} .
$$

Integrating Eq.(3.4) with respect to $r$, we can get

$$
\sigma_{r r}(r)+\int_{a}^{r} K(r, \rho) \sigma_{r r}(\rho) d \rho=D+C f(r)+F_{l}(r)
$$

with 


$$
\begin{aligned}
& K(r, \rho)=\frac{2}{\rho}\left(1-\frac{v(\rho)}{\lambda(\rho)}\right)-\frac{\left[\lambda(\rho)-2 v^{2}(\rho)\right] \chi(\rho)}{\lambda(\rho) E(\rho)} \int_{\rho}^{r} \frac{2 E^{*}(s)}{s^{2} \chi(s)[1-v(s)]} d s, \\
& f(r)=\int_{a}^{r} \frac{2 E^{*}(s)}{s^{2} \chi(s)[1-v(s)]} d s
\end{aligned}
$$

and

$$
F_{l}(r)=\int_{a}^{r} F(s) d s
$$

\section{Numerical results and discussions}

Defining normalized variables $x$ and $t$ such that

$$
r=\frac{(b+a)}{2}+\frac{(b-a) x}{2} \text { and } \rho=\frac{(b+a)}{2}+\frac{(b-a) t}{2},
$$

Eq.(2.19) can be rewritten as

$$
\sigma(x)+\int_{-1}^{l} G(x, t) \sigma(t) d t=g(x)
$$

where $\sigma(x)=\sigma_{r r}(r), g(x)=h(r)$ and $G(x, t)=\frac{(b-a) L(r, \rho)}{2}$.

In this case, to determine the numerical solution to Eq.(4.1), we invoke the Legendre polynomial method and $\sigma(x)$ can be expanded as

$$
\sigma(x)=\sum_{n=0}^{N} c_{n} P_{n}(x), \quad-1 \leq x \leq 1
$$

where $P_{n}(x)$ are Legendre polynomials, $c_{n}$ are unknown coefficients, and the first $\mathrm{N}+1$ terms are chosen, due to a negligible contribution of the rest.

It is easily shown that $c_{n}$ can be determined by solving a system of linear algebraic equations (Atkinson and Shampine [1]):

$$
\frac{2}{2 p+1} c_{p}+\sum l_{p n} c_{n}=g p, \quad p=0,1,2,3,---, N,
$$

with

$$
l_{p n}=\int_{-1}^{1} \int_{-1}^{1} P_{p}(x) F(x, t) P_{n}(t) d t d x
$$

and 


$$
g_{p}=\int_{-1}^{1} P_{p}(x) g(x) d x .
$$

Once $\sigma_{r r}(r)=\sigma(x)$ is obtained, $u_{r}$ and $\sigma_{\theta \theta}$ can be obtained from Eqs (2.12) and (2.14) respectively. For a transversely isotropic hollow sphere, $u_{r}$ and $\sigma_{\theta \theta}$ can be obtained by using a similar procedure.

For most materials, the Poisson's ratio is nearly a constant, so we only consider arbitrary radial nonhomogeneity of Young's modulus.

In order to show the effectiveness and accuracy of the present method, here we first compare our numerical results with existing analytical results for special gradient forms. For this, we consider the Young's modulus in power-law form: i.e.

$$
E(r)=E_{i}\left(\frac{r}{a}\right)^{m}, \quad v(r)=v
$$

where $E_{i}$ is the Young's modulus at the inner surface.

For this case, the exact stress distribution induced by internally uniform pressure $-q_{i}$ and $q_{0}=0$ as Guven and Baykara [3]:

$$
\begin{aligned}
& \sigma_{r r}(r)=-q_{i}\left(\frac{a}{r}\right)^{(3+k-m) / 2}\left(\frac{b^{k}-r^{k}}{b^{k}-a^{k}}\right)+ \\
& -\frac{\alpha_{0} E_{i}\left(T_{b}-T_{a}\right)}{1-v}\left(\frac{a}{r}\right)^{(3+k-m) / 2}\left[\frac{b^{k}-r^{k}}{b^{k}-a^{k}}-\left(\frac{a}{r}\right)^{(-1-k+m) / 2}\left(\frac{b-r}{b-a}\right)\right], \\
& \sigma_{\theta \theta}(r)=-q_{i}\left(\frac{a}{r}\right)^{(3+k-m) / 2} \frac{1}{b^{k}-a^{k}}\left[\frac{2-(1+m+k) v}{(1-v)(1+m+k)-4 v} b^{k}+\right. \\
& \left.-\frac{2-(1+m-k) v}{(1-v)(1+m-k)-4 v} r^{k}\right]-\frac{\alpha_{0} E_{i}\left(T_{a}-T_{b}\right)}{2(1-v)}\left(\frac{a}{r}\right)^{(3+k-m) / 2} \times \\
& \times\left[\frac{b^{k}+2 r^{k}}{b^{k}-a^{k}}+\left(\frac{a}{r}\right)^{(-1-k+m) / 2}\left(\frac{b-2 r}{b-a}\right)\right]
\end{aligned}
$$

where

$$
k=\sqrt{(1+m)^{2}+8-8 m v /(1-v)} .
$$

The numerical results of the radial and tangential stresses are evaluated for several different values of the gradient index $\mathrm{m}$ for $\frac{a}{b}=0.6, v=0.3, E_{i}=500 \mathrm{Gpa}, \alpha_{0}=10 \times 10^{-6}, q_{i}=500, T_{a}=0^{0} \mathrm{C}$ and $T_{b}=10^{\circ} \mathrm{C}$. 


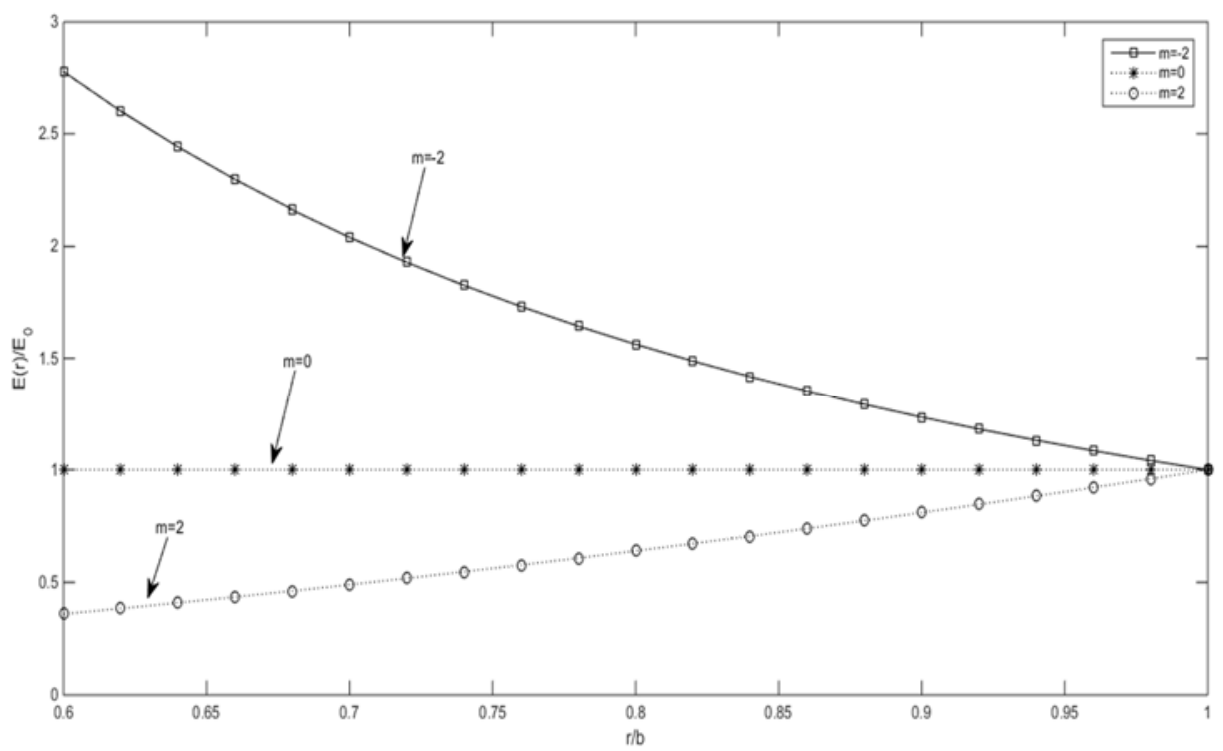

Fig.1. Variations of the elasticity modulus $E(r)$ in the radial direction.

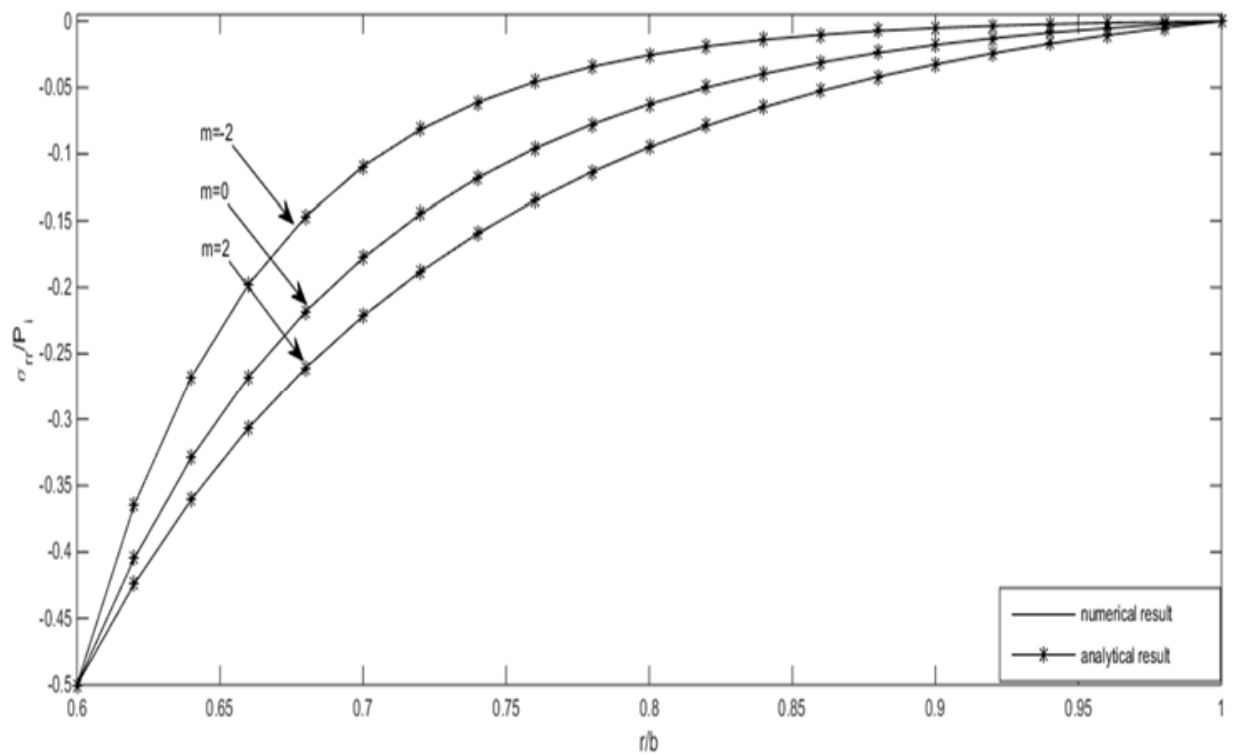

Fig.2. Variations of the radial stress $\frac{\sigma_{r r}(r)}{P_{i}}$ in the FGM hollow sphere with $a / b=0.6, v=0.3$ and $E(r)$ given by (4.4); numerical results (solid lines) with $N=3$ and analytical results (scattered line with asterisks) by Eqs (4.5) and (4.6).

Figure 1 illustrates variations of the elasticity modulus $E(r)$ in the radial direction of the sphere for $m=-2$, $m=0$ and $m=2$. For $m=0$, the elasticity modulus is a constant value in the radial direction. For $m=-2$ and $m=2$, the elasticity modulus decreases approximately linearly throughout, from outer surface to the inner surface depending on the function, $E(r)=E(r / b)^{m}$. These are used for analytical and numerical solutions. 
Figures 2 and 3 shows the distribution of $\frac{\sigma_{r r}(r)}{q_{i}}$ and $\frac{\sigma_{\theta \theta}(r)}{q_{i}}$. By taking only the first four terms of Legendre polynomials $(N=3)$, the obtained results are rather satisfactory. The solid lines correspond to the numerical results with $N=3$ and scattered lines with asterisks (analytical results) evaluated from the analytical expressions given by Eqs (4.5) and (4.6), are coincident. So, from Figs 2 and 3, it is seen that the numerical results are quite accurate, and so the method is very efficient.

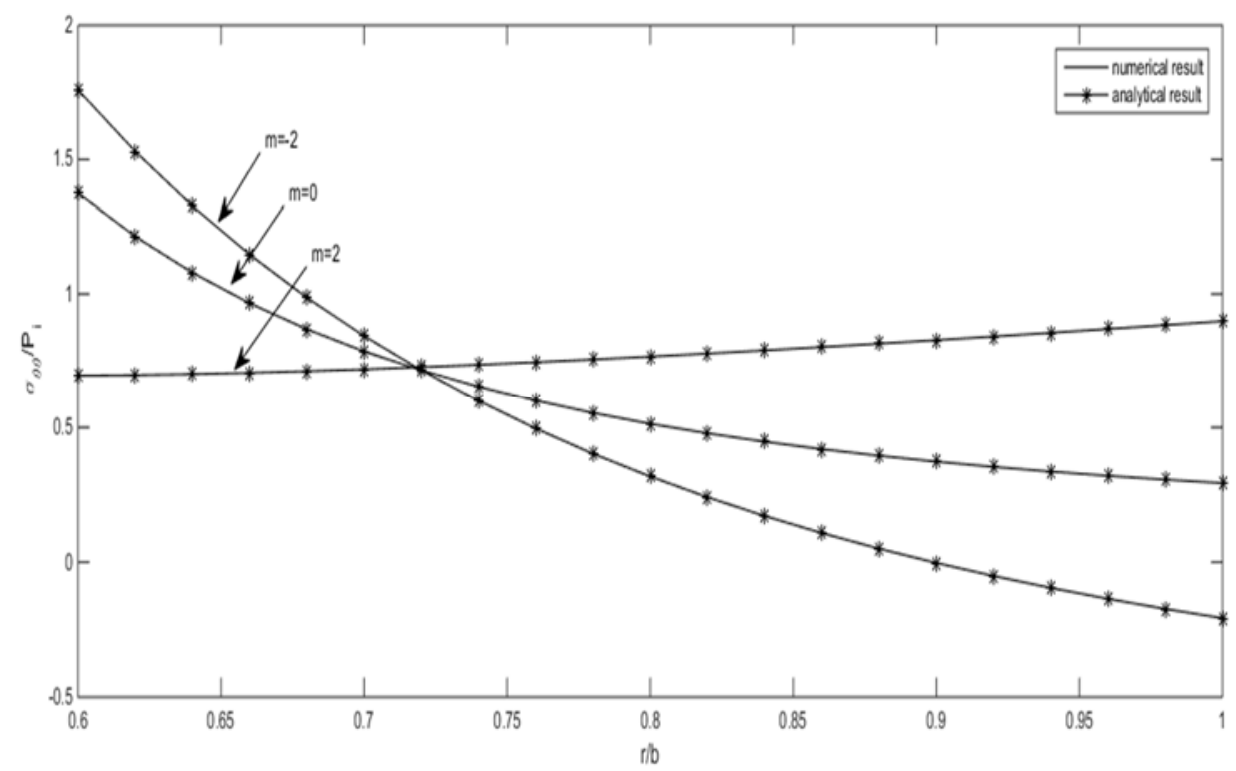

Fig.3. Variations of $\frac{\sigma_{\theta \theta}(r)}{P_{i}}$ in the FGM hollow sphere with $a / b=0.6, v=0.3$ and $E(r)$ given by Eq.(4.4); numerical results (solid lines) with $N=3$ and analytical results (scattered line with asterisks) by Eqs (4.5) and (4.6).

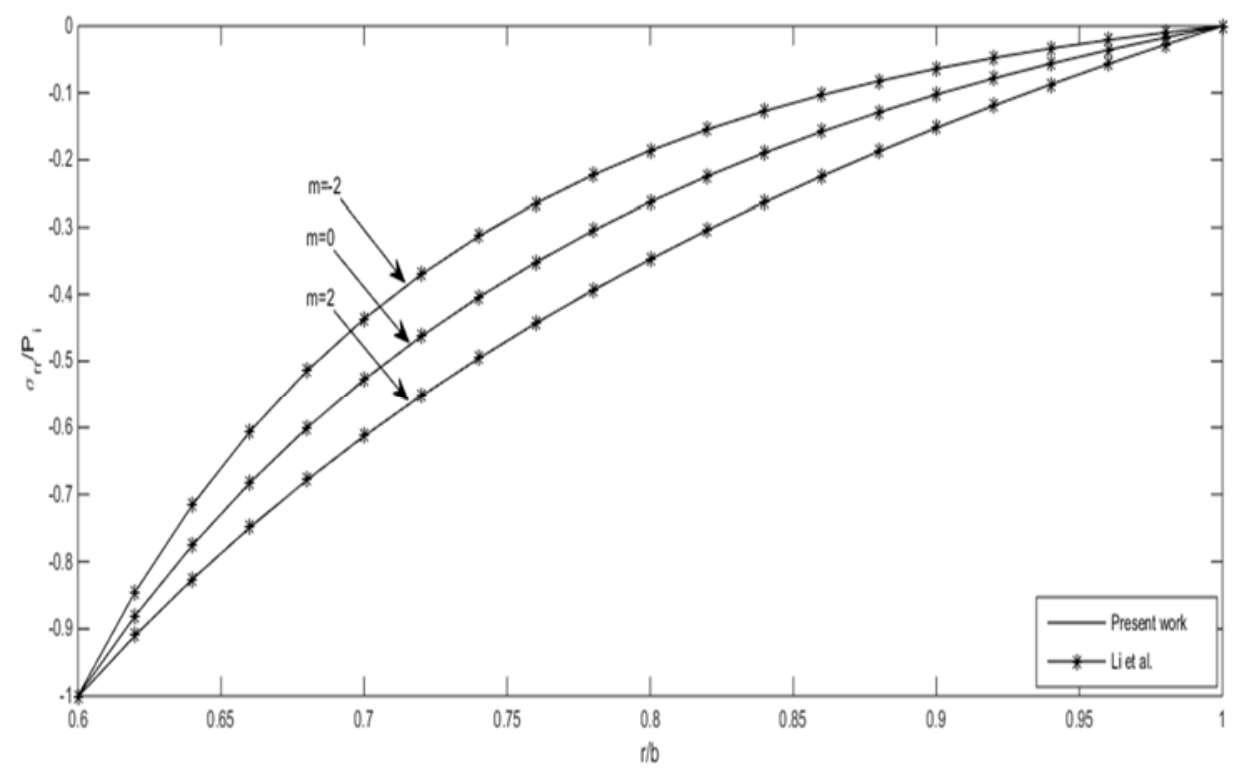

Fig.4. Variation of $\frac{\sigma_{r r}(r)}{P_{i}}$ with the radius $r$ of the sphere. 


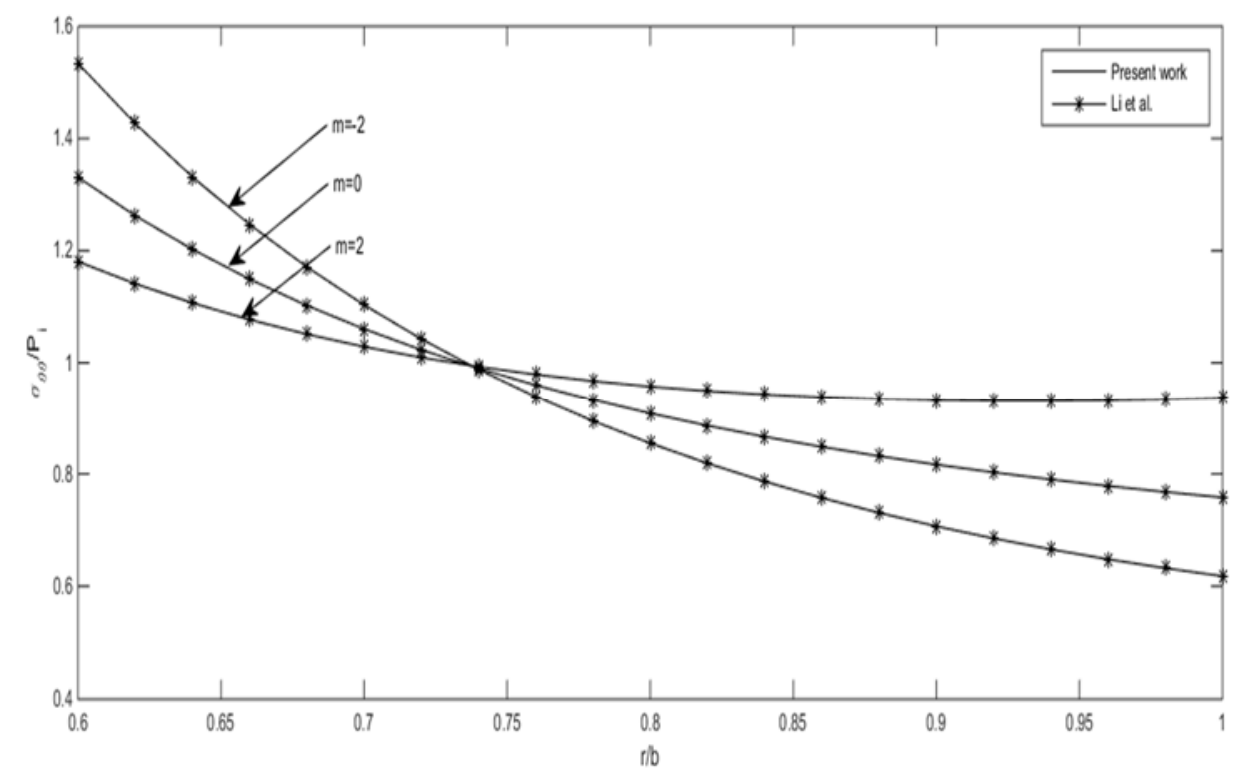

Fig.5. Variation of $\frac{\sigma_{\theta \theta}(r)}{P_{i}}$ with the radius $r$ of the sphere.

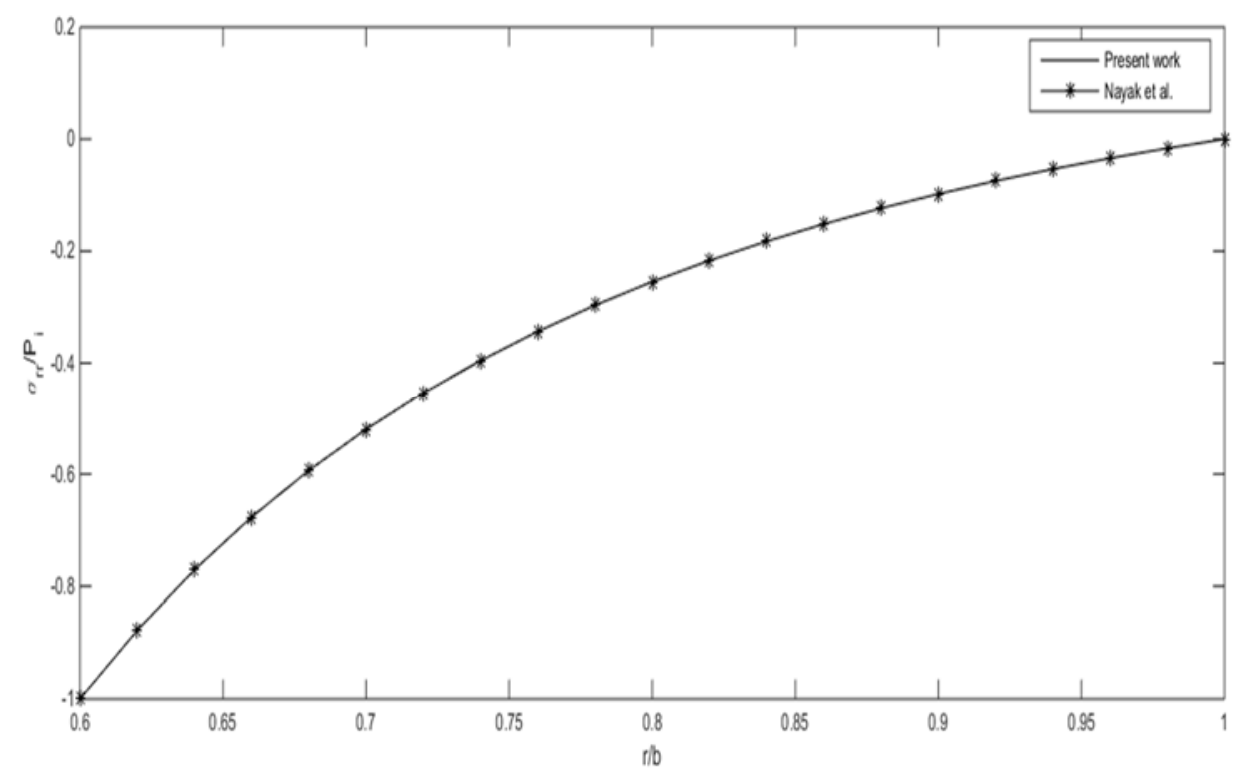

Fig.6. Variation of $\frac{\sigma_{\theta \theta}(r)}{P_{i}}$ with the radius $r$ of the sphere.

In Fig.2., variations of radial stresses $\sigma_{r r}(r)$ obtained from analytical and numerical solutions are shown in the radial direction for $m=-2,0$ and 2. These are zero at the outer surface but these are higher at radius $r \approx \frac{a+b}{2}$ for $m=-2$ and $m=2$ and $r=\sqrt{a b}$ for $m=0$.

In Fig.3., variations of tangential stresses $\sigma_{\theta \theta}(r)$ are shown in the radial direction for $m=-2 ; 0 ; 2$. In the FGM sphere, $\sigma_{\theta \theta}(r)$ reaches its maximum tensile stress at the inner surface, then progressively drops when $r$ rises. 
Validation of present work is carried out on the basis of results obtained by Li et al. [4] and comparison is presented in Figs 4 and 5. The results obtained are found to be in good agreement with Li et al. [4]. This establishes validity of the mathematical formulation and is further used for the investigation of the functionally graded hollow sphere by neglecting thermal effects from the present work with Li et al. [4].

If we take an isotropic homogeneous material (taking $m=0$ ) then the results obtained for thermal stresses are validated against the results of Nayak et al. [5]. A comparison is presented in Figs 6 and 7.

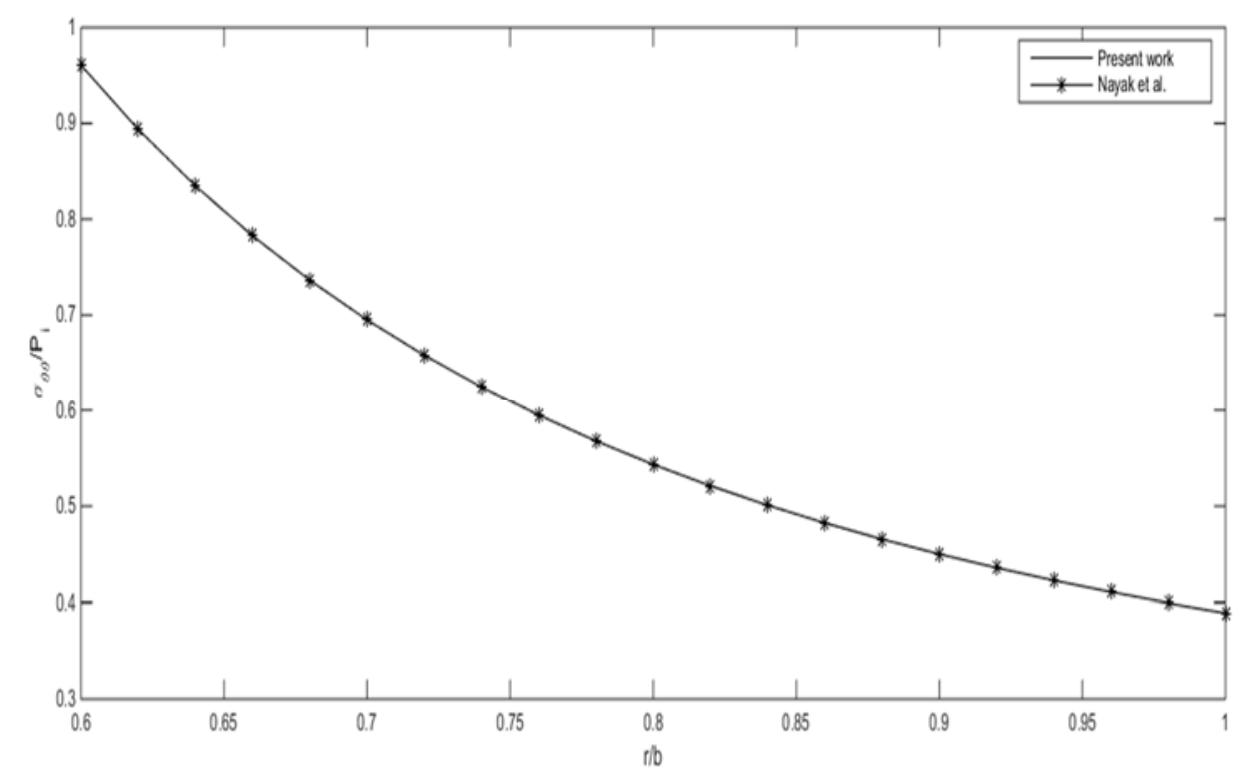

Fig.7. Variation of $\frac{\sigma_{\theta \theta}(r)}{P_{i}}$ with the radius $r$ of the sphere.

\section{Conclusion}

The symmetrical problem of an FGM sphere has been studied for spherical isotropy and spherical transversely isotropy. In this study, the thermoelastic problem is converted to a Fredholm integral equation. By solving the resulting equation, the distributions of the thermal stresses are obtained and presented graphically. The results obtained by this approach coincides with the closed-form analytical solution for the power-law gradient. The effects of the gradient variation of the material properties on the induced thermal stresses are discussed. Further, the obtained results coincide with Li et al. [4] by neglecting thermal effects and Nayak et al. [5] for isotropic homogeneous material.

\section{Nomenclature}

$$
\begin{aligned}
a & - \text { inner radius } \\
b & - \text { outer radius } \\
c_{p} & - \text { constant coefficients } \\
E, E(r), E^{*}(r) & - \text { Young's modulus (dependent on r) } \\
E_{i} & - \text { Young's modulus at the inner surface } \\
v, v(r) & - \text { Poisson's ratio (dependent on } \mathrm{r} \text { ) } \\
\sigma_{r r} & - \text { radial stress }
\end{aligned}
$$




$$
\begin{aligned}
& \sigma_{\theta \theta} \quad-\text { tangential stress } \\
& \alpha, \alpha(r) \quad \text { - thermal expansion coefficient (dependent on } \mathrm{r} \text { ) } \\
& T \text { - temperature change } \\
& T_{a} \text { - temperature at the inner surface } \\
& T_{b} \text { - temperature at the outer surface } \\
& A, B, C, D \text { - constants of integration } \\
& G(r, t) \text { - kernel of normalized Fredholm integral equation } \\
& f(r), F(r), F_{I}(r) \text { - known functions defined by Eq.(17) } \\
& K(r, t), L(r, t) \quad \text { - kernel of Fredholm integral equation } \\
& q_{i}, q_{0} \quad \text { - pressure at the inner and outer surface } \\
& r \text { - radial coordinate } \\
& x, t \quad \text { - normalized radial variables } \\
& u_{r} \text { - radial displacement } \\
& p \text { - gradient index } \\
& \theta \text { - polar angle coordinate }
\end{aligned}
$$

\section{REFERENCES}

[1] Atkinson K.E. and Shampine L.F. (2008): Algorithm 876: Solving Fredholm integral equations of the second kind in MATLAB.- ACM Transactions on Mathematical Software, vol.34, No.4, Article No.21, pp.1-20. Doi: https://doi.org/10.1145/1377596.137760134:21.

[2] Eslami M.R., Babaei M.H. and Poultangari R. (2005): Thermal and mechanical stresses in a functionally graded thick sphere.- Int. J. Pres. Ves. Pip., vol.82, pp.522-527.

[3] Guven U. and Baykara C. (2001): On stress distribution in functionally graded isotropic spheres subjected to internal pressure.- Mech. Res. Commun., vol.28, pp.277-281.

[4] Li X.F., Peng X.L. and Kang Y.A. (2009): Pressurized hollow spherical vessels with arbitrary radial nonhomogeneity.- AIAA Journal, vol.47, pp.2262-2265.

[5] Nayak P., Mondal S.C. and Nandi A. (2011): Stress, strain and displacement of a functionally graded thick spherical vessel.- Int. J. Eng. Sci. Techol., vol.3, pp.2659-2671.

[6] Nejad M.Z., Abedi M., Lotfian M.H. and Ghannad M. (2016): Exact and numerical elastic analysis for the FGM thickwalled cylindrical pressure vessels with exponentially-varying properties.- Arch. Metall. Mater., vol.61, pp.1649-1654.

[7] Peng X.L. and Li X.F. (2010): Thermoelastic analysis of a cylindrical vessel of functionally graded materials.- Int. J. Pres. Ves. Pip., vol.87, pp.203-210.

[8] Rani P., Singh K. and Muwal R. (2021): Thermal stresses due to non-uniform internal heat generation in functionally graded hollow cylinder.- Int. J. Appl. Mech. Eng., vol.6, pp.186-200.

[9] Sahu R.K., Sodhi L., Bhowmick S. and Thawait A.K. (2020): Exact solution for the thermo-elastic deformation and stress states of FG rotating spherical body.- J. Mech. Behav. Mater., vol.29, pp.133-146.

[10] Sharma D. and Kaur R. (2020): Thermoelastic analysis of FGM hollow cylinder for variable parameters and temperature distributions using FEM.- Nonlinear Eng., vol.9, pp.256-264.

[11] Yildirim V. (2018): Exact thermo-mechanical analysis of functionally graded thick-walled spheres.- Mech. Mech. Eng., vol.22, pp.1197-1221.

[12] You L.H., Zhang J.J. and You X.Y. (2005): Exact analysis of internally pressurized thick-walled spherical pressure vessels of functionally graded materials.- Int. J. Pres. Ves. Pip., vol.82, pp.347-354.

Received: June 17, 2021

Revised: $\quad$ September 21, 2021 